\title{
Accurate Asymptotic Formulas for Eigenvalues and Eigenfunctions of a Boundary-Value Problem of Fourth Order
}

\author{
Hamza Menken \\ Mathematics Department, Science and Arts Faculty, Mersin University, 33343 Mersin, Turkey \\ Correspondence should be addressed to Hamza Menken, hmenken@mersin.edu.tr \\ Received 7 July 2010; Accepted 9 November 2010 \\ Academic Editor: I. T. Kiguradze
}

Copyright (C) 2010 Hamza Menken. This is an open access article distributed under the Creative Commons Attribution License, which permits unrestricted use, distribution, and reproduction in any medium, provided the original work is properly cited.

In the present paper, we consider a nonself-adjoint fourth-order differential operator with the periodic boundary conditions. We compute new accurate asymptotic expression of the fundamental solutions of the given equation. Then, we obtain new accurate asymptotic formulas for eigenvalues and eigenfunctions.

\section{Introduction}

In the present work, we consider a nonself-adjoint fourth-order operator which is generated by the periodic boundary conditions:

$$
\begin{gathered}
y^{(4)}+q(x) y=\lambda y, \quad(0 \leq x \leq \pi), \\
y^{(j)}(0)-y^{(j)}(\pi)=0, \quad j=0,1,2,3,
\end{gathered}
$$

where $q(x)$ is a complex-valued function. Without lose of generality, we can assume that $\int_{0}^{\pi} q(x) d x=0$.

Spectral properties of Sturm-Liouville operator which is generated by the periodic and antiperiodic boundary conditions have been investigated by many authors, the results on this direct and references are given details in the monographs [1-5].

In this paper we obtain asymptotic formulas for the eigenvalues and eigenfunctions of the fourth-order boundary-value problem (1.1), (1.2). For second-order differential equations, similar asymptotic formulas were obtained in [6-9]. We note that in $[6,10,11]$, 
using the obtained asymptotic formulas for eigenvalues and eigenfunctions, the basis properties of the root functions of the operators were investigated.

The paper is organized as follows. In Section 2, we compute new asymptotic expression of the fundamental solutions of (1.1). In Section 3, we obtain new accurate asymptotic estimates for the eigenvalues. In Section 4 , we have asymptotic formulas for eigenfunctions under the distinct conditions on $q(x)$.

\section{The Expression of the Fundamental Solutions}

It is well known that (see [2, page 92]) if the complex $s$-plane $\left(s^{4}=\lambda\right)$ is divided into eight sectors $S_{\ell}(\ell=\overline{0,7})$, defined by the inequalities

$$
\frac{\ell \pi}{8} \leq \arg s \leq \frac{(\ell+1) \pi}{8}, \quad(\ell=\overline{0,7}),
$$

then in each of these sectors (1.1) has four linear independent solutions $y_{k}(x, s)(k=1,2,3,4)$, which are regular with respect to $s$ in the sector $S_{l}$ for $|s|$ sufficiently large and which satisfy the relation

$$
y_{k}(x, s)=e^{\omega_{k} s x}\left[\sum_{v=0}^{7} \frac{u_{v, k}(x)}{s^{v}}+O\left(\frac{1}{s^{8}}\right)\right], \quad(k=1,2,3,4),
$$

where the numbers $\omega_{k}$ are the fourth roots of unity, that is, $\omega_{1}=-\omega_{4}=i$ and $\omega_{2}=-\omega_{3}=1$. In general, the term $O\left(s^{-(N+1)}\right)$ at the formula (2.2) depends upon the smoothness of the function $q(x)$. If $q(x)$ has $m$ continuous derivatives, then one can assert the existence of a representation (2.2) with $N=m+3$. Here, we assume that $q(x) \in C^{(4)}[0, \pi]$. The functions $u_{v, k}(x)$ satisfy the following recursion relations:

$$
\begin{aligned}
& 4 u_{v, k}^{\prime}(x)+6 \omega_{k}^{3} u_{v-1, k}^{\prime \prime}(x)+4 \omega_{k}^{2} u_{v-2, k}^{\prime \prime \prime}(x) \\
& +\omega_{k} u_{v-3, k}^{(4)}(x)+\omega_{k} q(x) u_{v-3, k}(x)=0, \quad u_{v, k}(x) \equiv 0, v<0 .
\end{aligned}
$$

Let us put, moreover, $u_{0, k}(x) \equiv 1, u_{v, k}(0) \equiv 0$, for $v \geq 1$. Thus, the functions $u_{v, k}(x)$ are uniquely determined. Thus, we can find from (2.3) that

$$
\begin{gathered}
u_{0, k}(x)=1, \quad u_{1, k}(x)=0, \quad u_{2, k}(x)=0, \quad u_{3, k}(x)=-\frac{1}{4 \omega_{k}^{3}} \int_{0}^{x} q(t) d t \\
u_{4, k}(x)=\frac{3}{8}[q(x)-q(0)], \quad u_{5, k}(x)=-\frac{5 \omega_{k}^{3}}{16}\left[q^{\prime}(x)-q^{\prime}(0)\right] \\
u_{6, k}(x)=\frac{3 \omega_{k}^{2}}{32}\left[q^{\prime \prime}(x)-q^{\prime \prime}(0)\right]+\frac{\omega_{k}^{2}}{32}\left(\int_{0}^{x} q(t) d t\right)^{2}, \\
u_{7, k}(x)=-\frac{\omega_{k}}{64}\left[q^{\prime \prime \prime}(x)-q^{\prime \prime \prime}(0)\right]-\frac{3 \omega_{k}}{32}[q(x)-q(0)] \int_{0}^{x} q(t) d t-\frac{3 \omega_{k}}{32} \int_{0}^{x} q^{2}(t) d t .
\end{gathered}
$$




\section{The Asymptotic Formulas of Eigenvalues}

It follows from the classical investigations (see [4, page 65]) that the eigenvalues of the problem (1.1), (1.2) (in $[0,1]$ ) consist of the pairs of the sequences $\left\{\lambda_{k, 1}\right\},\left\{\lambda_{k, 2}\right\}$ satisfying the following asymptotic formula:

$$
\lambda_{k, 1}=\lambda_{k, 2}+O\left(k^{1 / 2}\right)=(2 k \pi)^{4}\left(1+\frac{\xi_{0}}{k}+O\left(\frac{1}{k^{3 / 2}}\right)\right)
$$

for sufficiently large integer $k$, where $\xi_{0}$ is a constant.

Theorem 3.1. Assume that $q(x) \in C^{(4)}[0, \pi]$. Then, the eigenvalues of the boundary-value problem (1.1), (1.2) form two infinite sequences $\lambda_{k, 1}, \lambda_{k, 2}(k=N, N+1, \ldots)$, where $N$ is a big positive integer and have the following asymptotic formulas:

$$
\begin{aligned}
& \lambda_{k, 1}=(2 k i)^{4}+\frac{3}{8 \pi} \frac{\int_{0}^{\pi} q^{2}(t) d t}{(2 k i)^{4}}+O\left(\frac{1}{k^{8}}\right), \\
& \lambda_{k, 2}=(2 k)^{4}+\frac{3}{8 \pi} \frac{\int_{0}^{\pi} q^{2}(t) d t}{(2 k)^{4}}+O\left(\frac{1}{k^{8}}\right) .
\end{aligned}
$$

Proof. By derivation of (2.2) up to third order with respect to $x$, the following relations are obtained:

$$
y_{k}^{(m)}(x, s)=\left(\omega_{k} s\right)^{m} e^{\omega_{k} s x}\left[\sum_{v=0}^{7} \frac{u_{v, k}^{(m)}(x)}{s^{v}}+O\left(\frac{1}{s^{8}}\right)\right],
$$

where $k=1,2,3,4, m=1,2,3$ and

$$
\begin{gathered}
u_{0, k}^{(m)}(x)=1, \quad u_{1, k}^{(m)}(x)=0, \quad u_{2, k}^{(m)}(x)=0, \quad u_{3, k}^{(m)}(x)=-\frac{1}{4 \omega_{k}^{3}} \int_{0}^{x} q(t) d t, \\
u_{4, k}^{(1)}(x)=\frac{1}{8} q(x)-\frac{3}{8} q(0), \quad u_{4, k}^{(2)}(x)=-\frac{1}{8} q(x)-\frac{3}{8} q(0), \quad u_{4, k}^{(3)}(x)=-\frac{3}{8} q(x)-\frac{3}{8} q(0), \\
u_{5, k}^{(1)}(x)=\frac{\omega_{k}^{3}}{16} q^{\prime}(x)+\frac{5 \omega_{k}^{3}}{16} q^{\prime}(0), \quad u_{5, k}^{(2)}(x)=\frac{3 \omega_{k}^{3}}{16} q^{\prime}(x)+\frac{5 \omega_{k}^{3}}{16} q^{\prime}(0), \\
u_{5, k}^{(3)}(x)=\frac{\omega_{k}^{3}}{16} q^{\prime}(x)+\frac{5 \omega_{k}^{3}}{16} q^{\prime}(0),
\end{gathered}
$$




$$
\begin{gathered}
u_{6, k}^{(1)}(x)=-\frac{5 \omega_{k}^{2}}{32}\left[q^{\prime \prime}(x)+q^{\prime \prime}(0)\right]+\frac{\omega_{k}^{2}}{32}\left(\int_{0}^{x} q(t) d t\right)^{2}, \\
u_{6, k}^{(2)}(x)=-\frac{3 \omega_{k}^{2}}{32} q^{\prime \prime}(x)-\frac{5 \omega_{k}^{2}}{32} q^{\prime \prime}(0)+\frac{\omega_{k}^{2}}{32}\left(\int_{0}^{x} q(t) d t\right)^{2}, \\
u_{6, k}^{(3)}(x)=\frac{3 \omega_{k}^{2}}{32} q^{\prime \prime}(x)-\frac{5 \omega_{k}^{2}}{32} q^{\prime \prime}(0)+\frac{\omega_{k}^{2}}{32}\left(\int_{0}^{x} q(t) d t\right)^{2}, \\
u_{7, k}^{(1)}(x)=\frac{9 \omega_{k}}{64} q^{\prime \prime \prime}(x)+\frac{\omega_{k}}{64} q^{\prime \prime \prime}(0)-\frac{\omega_{k}}{32}[q(x)-3 q(0)] \int_{0}^{x} q(t) d t-\frac{3 \omega_{k}}{32} \int_{0}^{x} q^{2}(t) d t, \\
u_{7, k}^{(2)}(x)=-\frac{\omega_{k}}{64} q^{\prime \prime \prime}(x)+\frac{\omega_{k}}{64} q^{\prime \prime \prime}(0)+\frac{\omega_{k}}{32}[q(x)+3 q(0)] \int_{0}^{x} q(t) d t-\frac{3 \omega_{k}}{32} \int_{0}^{x} q^{2}(t) d t, \\
u_{7, k}^{(3)}(x)=-\frac{7 \omega_{k}}{64} q^{\prime \prime \prime}(x)+\frac{\omega_{k}}{64} q^{\prime \prime \prime}(0)+\frac{3 \omega_{k}}{32}[q(x)+3 q(0)] \int_{0}^{x} q(t) d t-\frac{3 \omega_{k}}{32} \int_{0}^{x} q^{2}(t) d t .
\end{gathered}
$$

Now let us substitute all these expressions into the characteristic determinant

$$
\Delta(s)=\left|\begin{array}{llll}
U_{1}\left(y_{1}\right) & U_{1}\left(y_{2}\right) & U_{1}\left(y_{3}\right) & U_{1}\left(y_{4}\right) \\
U_{2}\left(y_{1}\right) & U_{2}\left(y_{2}\right) & U_{2}\left(y_{3}\right) & U_{2}\left(y_{4}\right) \\
U_{3}\left(y_{1}\right) & U_{3}\left(y_{2}\right) & U_{3}\left(y_{3}\right) & U_{3}\left(y_{4}\right) \\
U_{4}\left(y_{1}\right) & U_{4}\left(y_{2}\right) & U_{4}\left(y_{3}\right) & U_{4}\left(y_{4}\right)
\end{array}\right|,
$$

where

$$
U_{j+1}\left(y_{k}\right)=y_{k}^{(j)}(\pi)-y_{k}^{(j)}(0), \quad(j=0,1,2,3)
$$

By long computations, for sufficiently large $|s|$, we obtain that

$$
\begin{aligned}
\Delta(s)= & 16 i s^{6}\left\{e^{s \pi}\left[1-\frac{3}{2} \frac{q(0)}{s^{4}}-\frac{3}{32} \frac{\int_{0}^{\pi} q^{2}(t) d t}{s^{7}}+O\left(\frac{1}{s^{8}}\right)\right]\right. \\
& \left.-2\left[1-\frac{3}{2} \frac{q(0)}{s^{4}}+O\left(\frac{1}{s^{8}}\right)\right]+e^{-s \pi}\left[1-\frac{3}{2} \frac{q(0)}{s^{4}}+\frac{3}{32} \frac{\int_{0}^{\pi} q^{2}(t) d t}{s^{7}}+O\left(\frac{1}{s^{8}}\right)\right]\right\} \\
& \times\left\{e ^ { i s \pi } \left[1-\frac{3 i}{32} \frac{\left.\int_{0}^{\pi} \frac{q^{2}(t) d t}{s^{7}}+O\left(\frac{1}{s^{8}}\right)\right]-2\left[1+O\left(\frac{1}{s^{8}}\right)\right]}{+}\right.\right. \\
& \left.e^{-i s \pi}\left[1+\frac{3 i}{32} \frac{\int_{0}^{\pi} q^{2}(t) d t}{s^{7}}+O\left(\frac{1}{s^{8}}\right)\right]\right\} .
\end{aligned}
$$


Multiplying the last equation by

$$
e^{s \pi}\left[1+\frac{3}{2} \frac{q(0)}{s^{4}}+\frac{3}{32} \frac{\int_{0}^{\pi} q^{2}(t) d t}{s^{7}}+O\left(\frac{1}{s^{8}}\right)\right] e^{i s \pi}\left[1+\frac{3 i}{32} \frac{\int_{0}^{\pi} q^{2}(t) d t}{s^{7}}+O\left(\frac{1}{s^{8}}\right)\right]
$$

it becomes

$$
\left\{e^{s \pi}-\left[1+\frac{3}{32} \frac{\int_{0}^{\pi} q^{2}(t) d t}{s^{7}}+O\left(\frac{1}{s^{8}}\right)\right]\right\}^{2}\left\{e^{i s \pi}-\left[1+\frac{3 i}{32} \frac{\int_{0}^{\pi} q^{2}(t) d t}{s^{7}}+O\left(\frac{1}{s^{8}}\right)\right]\right\}^{2} .
$$

Hence, by $\Delta(s)=0$, for sufficiently large $|s|$, the following equations hold:

$$
\begin{aligned}
& e^{s \pi}-1=\frac{3}{32} \frac{\int_{0}^{\pi} q^{2}(t) d t}{s^{7}}+O\left(\frac{1}{s^{8}}\right), \\
& e^{i s \pi}-1=\frac{3 i}{32} \frac{\int_{0}^{\pi} q^{2}(t) d t}{s^{7}}+O\left(\frac{1}{s^{8}}\right) .
\end{aligned}
$$

By Rouche's theorem, we have asymptotic estimates for the roots $s_{k, 1}$ and $s_{k, 2}, k=N, N+$ $1, \ldots$, of (3.10) and (3.11), respectively, where $N$ is a big positive integer

$$
\begin{aligned}
& s_{k, 1}=2 k i+\frac{3}{32 \pi} \frac{\int_{0}^{\pi} q^{2}(t) d t}{(2 k i)^{7}}+O\left(\frac{1}{k^{8}}\right), \\
& s_{k, 2}=2 k+\frac{3}{32 \pi} \frac{\int_{0}^{\pi} q^{2}(t) d t}{(2 k)^{7}}+O\left(\frac{1}{k^{8}}\right) .
\end{aligned}
$$

From the relations (3.12), (3.13) and the relations $\lambda_{k, j}=s_{k, j^{\prime}}^{4}(j=1,2)$, the asymptotic formulas (3.2) are valid for $k \geq N$.

\section{The Asymptotic Formulas for the Eigenfunctions}

Now, we obtain asymptotic formulas for eigenfunctions under the distinct conditions on $q(x)$.

Case 1. Assume that $q(x) \in C^{(1)}[0, \pi]$ and the condition $q(\pi)-q(0) \neq 0$ holds. Based on the asymptotic expressions of the fundamental solutions of (1.1) and the asymptotic formulas for eigenvalues of the boundary-value problem (1.1), (1.2) up to order $O\left(s^{-5}\right)$, the following result is valid. 
Theorem 4.1. If the condition $q(\pi)-q(0) \neq 0$ holds, then eigenfunctions of the boundary-value problem (1.1), (1.2) corresponding the eigenvalues $\lambda_{k, 1}$ and $\lambda_{k, 2}$ are of the form

$$
\begin{aligned}
& y_{k, 1}(x)=\sin (2 k x)-\sinh (2 k x)+O\left(\frac{1}{k}\right), \\
& y_{k, 2}(x)=\cos (2 k x)+\cosh (2 k x)+O\left(\frac{1}{k}\right),
\end{aligned}
$$

where $k$ is sufficiently large integer.

Proof. Let us calculate $U_{j+1}\left(y_{v}\left(x, s_{k, 1}\right)\right)(j=0,1,2)$ up to order $O\left(s_{k, 1}^{-5}\right)$. Since

$$
e^{s_{k, 1} \pi}-1=\frac{3}{32} \frac{\int_{0}^{\pi} q^{2}(t) d t}{s_{k, 1}^{7}}+O\left(\frac{1}{s_{k, 1}^{8}}\right)
$$

we obtain that

$$
\begin{gathered}
U_{1}\left(y_{v}\left(x, s_{k, 1}\right)\right)=\frac{3}{8} \frac{q(\pi)-q(0)}{s_{k, 1}^{4}}+O\left(\frac{1}{s_{k, 1}^{5}}\right), \\
U_{2}\left(y_{v}\left(x, s_{k, 1}\right)\right)=\omega_{v} s_{k, 1}\left[\frac{1}{8} \frac{q(\pi)-q(0)}{s_{k, 1}^{4}}+O\left(\frac{1}{s_{k, 1}^{5}}\right)\right] \\
U_{3}\left(y_{v}\left(x, s_{k, 1}\right)\right)=\left(\omega_{v} s_{k, 1}\right)^{2}\left[-\frac{1}{8} \frac{q(\pi)-q(0)}{s_{k, 1}^{4}}+O\left(\frac{1}{s_{k, 1}^{5}}\right)\right] .
\end{gathered}
$$

Follows from the condition $q(\pi)-q(0) \neq 0$ that $U_{j+1}\left(y_{v}\left(x, s_{k, 1}\right)\right) \neq 0$ for $j=0,1,2$. Thus, we can seek eigenfunction $y_{k, 1}(x)$ corresponding $\lambda_{k, 1}$ in the form

$$
y_{k, 1}(x)=\left|\begin{array}{cccc}
y_{1}\left(x, s_{k, 1}\right) & y_{2}\left(x, s_{k, 1}\right) & y_{3}\left(x, s_{k, 1}\right) & y_{4}\left(x, s_{k, 1}\right) \\
U_{1}\left(y_{1}\right) & U_{1}\left(y_{2}\right) & U_{1}\left(y_{3}\right) & U_{1}\left(y_{4}\right) \\
U_{2}\left(y_{1}\right) & U_{2}\left(y_{2}\right) & U_{2}\left(y_{3}\right) & U_{2}\left(y_{4}\right) \\
U_{3}\left(y_{1}\right) & U_{3}\left(y_{2}\right) & U_{3}\left(y_{3}\right) & U_{3}\left(y_{4}\right)
\end{array}\right| .
$$

Then,

$$
\begin{aligned}
y_{k, 1}(x)= & y_{1}\left(x, s_{k, 1}\right)\left|\begin{array}{lll}
U_{1}\left(y_{2}\right) & U_{1}\left(y_{3}\right) & U_{1}\left(y_{4}\right) \\
U_{2}\left(y_{2}\right) & U_{2}\left(y_{3}\right) & U_{2}\left(y_{4}\right) \\
U_{3}\left(y_{2}\right) & U_{3}\left(y_{3}\right) & U_{3}\left(y_{4}\right)
\end{array}\right| \\
& -y_{2}\left(x, s_{k, 1}\right)\left|\begin{array}{lll}
U_{1}\left(y_{1}\right) & U_{1}\left(y_{3}\right) & U_{1}\left(y_{4}\right) \\
U_{2}\left(y_{1}\right) & U_{2}\left(y_{3}\right) & U_{2}\left(y_{4}\right) \\
U_{3}\left(y_{1}\right) & U_{3}\left(y_{3}\right) & U_{3}\left(y_{4}\right)
\end{array}\right|
\end{aligned}
$$


Boundary Value Problems

$$
\begin{gathered}
+y_{3}\left(x, s_{k, 1}\right)\left|\begin{array}{lll}
U_{1}\left(y_{1}\right) & U_{1}\left(y_{2}\right) & U_{1}\left(y_{4}\right) \\
U_{2}\left(y_{1}\right) & U_{2}\left(y_{2}\right) & U_{2}\left(y_{4}\right) \\
U_{3}\left(y_{1}\right) & U_{3}\left(y_{2}\right) & U_{3}\left(y_{4}\right)
\end{array}\right| \\
-y_{4}\left(x, s_{k, 1}\right)\left|\begin{array}{lll}
U_{1}\left(y_{1}\right) & U_{1}\left(y_{2}\right) & U_{1}\left(y_{3}\right) \\
U_{2}\left(y_{1}\right) & U_{2}\left(y_{2}\right) & U_{2}\left(y_{3}\right) \\
U_{3}\left(y_{1}\right) & U_{3}\left(y_{2}\right) & U_{3}\left(y_{3}\right)
\end{array}\right| .
\end{gathered}
$$

By simple computations, we obtain

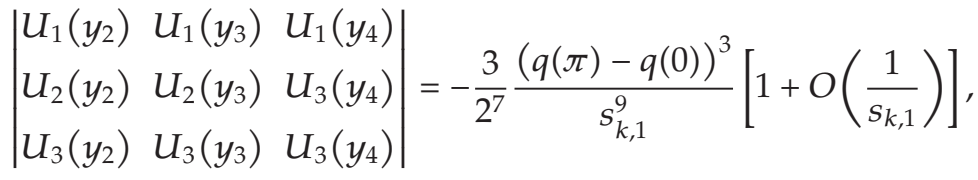

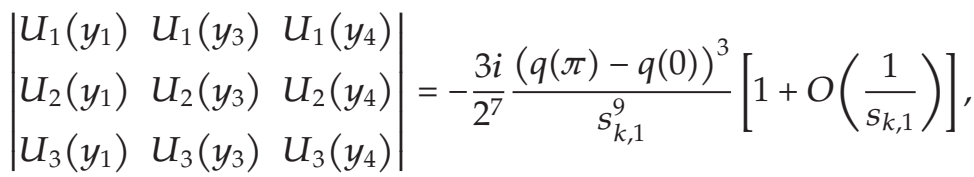

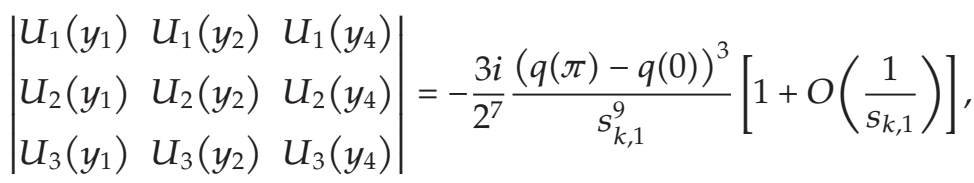

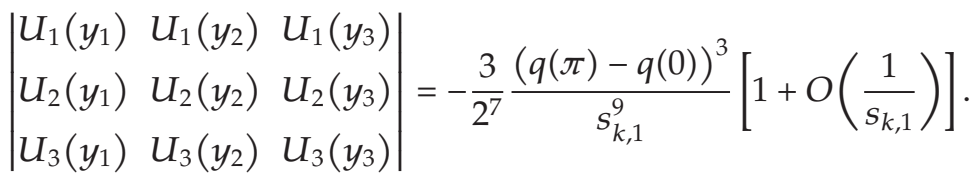

Hence, using the formula (2.2), we can write

$$
\begin{aligned}
y_{k, 1}(x) & =-\frac{3}{2^{7}} \frac{(q(\pi)-q(0))^{3}}{s_{k, 1}^{9}}\left[e^{i s_{k, 1} x}-i e^{s_{k, 1} x}+i e^{-s_{k, 1} x}-e^{-i s_{k, 1} x}+O\left(\frac{1}{s_{k, 1}}\right)\right] \\
& =-\frac{3}{2^{7}} \frac{(q(\pi)-q(0))^{3}}{s_{k, 1}^{9}}\left[2 i \sin s_{k, 1} x-2 i \sinh s_{k, 1} x+O\left(\frac{1}{s_{k, 1}}\right)\right] \\
& =-\frac{3 i}{2^{6}} \frac{(q(\pi)-q(0))^{3}}{s_{k, 1}^{9}}\left[\sin s_{k, 1} x-\sinh s_{k, 1} x+O\left(\frac{1}{s_{k, 1}}\right)\right] .
\end{aligned}
$$

Therefore, for the normalized eigenfunction, we get

$$
y_{k, 1}(x)=\sin s_{k, 1} x-\sinh s_{k, 1} x+O\left(\frac{1}{s_{k, 1}}\right) .
$$


Using the relations (3.3) and (3.12), for sufficiently large integer $k$, we obtain (4.1)

$$
y_{k, 1}(x)=\sin (2 k x)-\sinh (2 k x)+O\left(\frac{1}{k}\right) .
$$

Similarly, since $U_{j+1}\left(y_{v}\left(x, s_{k, 1}\right)\right) \neq 0$ for $j=1,2,3$, we can seek eigenfunction $y_{k, 2}(x)$ corresponding $\lambda_{k, 2}$ in the form

$$
y_{k, 2}(x)=\left|\begin{array}{cccc}
y_{1}\left(x, s_{k, 2}\right) & y_{2}\left(x, s_{k, 2}\right) & y_{3}\left(x, s_{k, 2}\right) & y_{4}\left(x, s_{k, 2}\right) \\
U_{2}\left(y_{1}\right) & U_{2}\left(y_{2}\right) & U_{2}\left(y_{3}\right) & U_{2}\left(y_{4}\right) \\
U_{3}\left(y_{1}\right) & U_{3}\left(y_{2}\right) & U_{3}\left(y_{3}\right) & U_{3}\left(y_{4}\right) \\
U_{4}\left(y_{1}\right) & U_{4}\left(y_{2}\right) & U_{4}\left(y_{3}\right) & U_{4}\left(y_{4}\right)
\end{array}\right| .
$$

Then,

$$
\begin{aligned}
y_{k, 2}(x)= & y_{1}\left(x, s_{k, 2}\right)\left|\begin{array}{lll}
U_{2}\left(y_{2}\right) & U_{2}\left(y_{3}\right) & u_{2}\left(y_{4}\right) \\
U_{3}\left(y_{2}\right) & U_{3}\left(y_{3}\right) & u_{3}\left(y_{4}\right) \\
U_{4}\left(y_{2}\right) & U_{4}\left(y_{3}\right) & U_{4}\left(y_{4}\right)
\end{array}\right| \\
& -y_{2}\left(x, s_{k, 2}\right)\left|\begin{array}{lll}
U_{2}\left(y_{1}\right) & U_{2}\left(y_{3}\right) & U_{2}\left(y_{4}\right) \\
U_{3}\left(y_{1}\right) & U_{3}\left(y_{3}\right) & U_{3}\left(y_{4}\right) \\
U_{4}\left(y_{1}\right) & U_{4}\left(y_{3}\right) & U_{4}\left(y_{4}\right)
\end{array}\right| \\
& +y_{3}\left(x, s_{k, 2}\right)\left|\begin{array}{lll}
U_{2}\left(y_{1}\right) & U_{2}\left(y_{2}\right) & U_{2}\left(y_{4}\right) \\
U_{3}\left(y_{1}\right) & U_{3}\left(y_{2}\right) & U_{3}\left(y_{4}\right) \\
U_{4}\left(y_{1}\right) & U_{4}\left(y_{2}\right) & U_{4}\left(y_{4}\right)
\end{array}\right| \\
& -y_{4}\left(x, s_{k, 2}\right)\left|\begin{array}{lll}
U_{2}\left(y_{1}\right) & U_{2}\left(y_{2}\right) & U_{2}\left(y_{3}\right) \\
U_{3}\left(y_{1}\right) & U_{3}\left(y_{2}\right) & U_{3}\left(y_{3}\right) \\
U_{4}\left(y_{1}\right) & U_{4}\left(y_{2}\right) & U_{4}\left(y_{3}\right)
\end{array}\right| .
\end{aligned}
$$

By similar computations we obtain

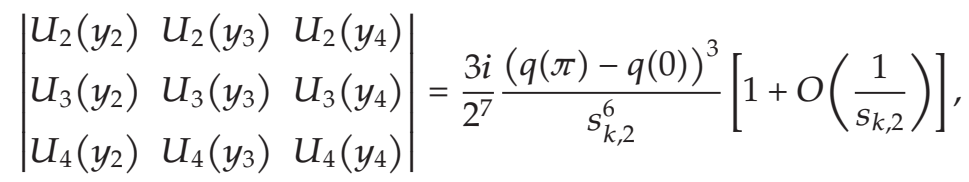

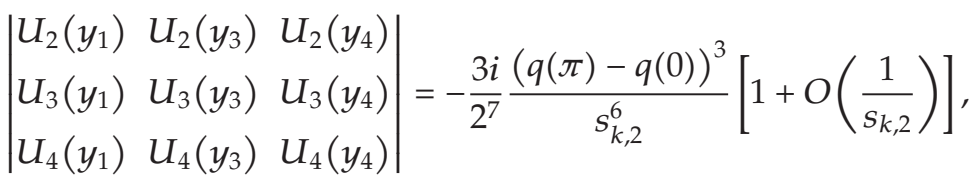




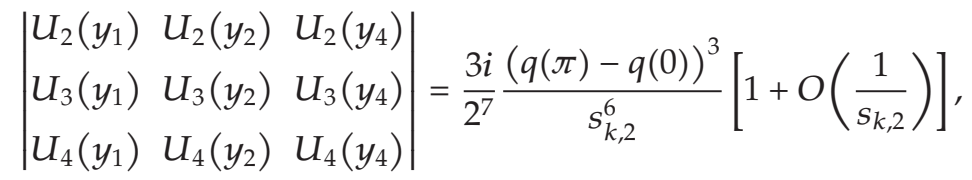

$$
\begin{aligned}
& \left|\begin{array}{lll}
U_{2}\left(y_{1}\right) & U_{2}\left(y_{2}\right) & U_{2}\left(y_{3}\right) \\
U_{3}\left(y_{1}\right) & U_{3}\left(y_{2}\right) & U_{3}\left(y_{3}\right) \\
U_{4}\left(y_{1}\right) & U_{4}\left(y_{2}\right) & U_{4}\left(y_{3}\right)
\end{array}\right|=-\frac{3 i}{2^{7}} \frac{(q(\pi)-q(0))^{3}}{s_{k, 2}^{6}}\left[1+O\left(\frac{1}{s_{k, 2}}\right)\right] .
\end{aligned}
$$

Hence, using the formula (2.2), we can write

$$
\begin{aligned}
y_{k, 2}(x) & =\frac{3 i}{2^{7}} \frac{(q(\pi)-q(0))^{3}}{s_{k, 2}^{6}}\left[e^{i s_{k, 2} x}+e^{s_{k, 2} x}+e^{-s_{k, 2} x}+e^{-i s_{k, 2} x}+O\left(\frac{1}{s_{k, 2}}\right)\right] \\
& =\frac{3 i}{2^{7}} \frac{(q(\pi)-q(0))^{3}}{s_{k, 2}^{6}}\left[2 \cos s_{k, 2} x+2 \cosh s_{k, 2} x+O\left(\frac{1}{s_{k, 2}}\right)\right] \\
& =\frac{3 i}{2^{6}} \frac{(q(\pi)-q(0))^{3}}{s_{k, 1}^{6}}\left[\cos s_{k, 2} x+\cosh s_{k, 2} x+O\left(\frac{1}{s_{k, 2}}\right)\right] .
\end{aligned}
$$

Therefore, for the normalized eigenfunction, we get

$$
y_{k, 2}(x)=\cos s_{k, 2} x+\cosh s_{k, 2} x+O\left(\frac{1}{s_{k, 2}}\right) .
$$

Hence, for sufficiently large integer $k$, we obtain (4.2)

$$
y_{k, 2}(x)=\cos (2 k x)+\cosh (2 k x)+O\left(\frac{1}{k}\right) .
$$

Case 2. Assume that $q(x) \in C^{(2)}[0, \pi]$ and the conditions $q(\pi)-q(0)=0$ and $q^{\prime}(\pi)-q^{\prime}(0) \neq 0$ hold. Based on the asymptotic expressions of the fundamental solutions of (1.1) and the asymptotic formulas for eigenvalues of the boundary-value problem (1.1), (1.2) up to order $O\left(s^{-6}\right)$, the following result is valid.

Theorem 4.2. If the conditions $q(\pi)-q(0)=0$ and $q^{\prime}(\pi)-q^{\prime}(0) \neq 0$ hold, then eigenfunctions of the boundary-value problem (1.1), (1.2) corresponding the eigenvalues $\lambda_{k, 1}$ and $\lambda_{k, 2}$ are of the form

$$
\begin{aligned}
& y_{k, 1}(x)=\cos (2 k x)-\cosh (2 k x)+O\left(\frac{1}{k}\right), \\
& y_{k, 2}(x)=\sin (2 k x)-\sinh (2 k x)+O\left(\frac{1}{k}\right),
\end{aligned}
$$

where $k$ is sufficiently large integer. 
Proof. It is clear that

$$
\begin{aligned}
& U_{1}\left(y_{v}\left(x, s_{k, 1}\right)\right)=\frac{3}{8} \frac{q(\pi)-q(0)}{s_{k, 1}^{4}}+\frac{5 \omega_{v}}{16} \frac{q^{\prime}(\pi)-q^{\prime}(0)}{s_{k, 1}^{5}}+O\left(\frac{1}{s_{k, 1}^{6}}\right), \\
& U_{2}\left(y_{v}\left(x, s_{k, 1}\right)\right)=\omega_{v} s_{k, 1}\left[\frac{1}{8} \frac{q(\pi)-q(0)}{s_{k, 1}^{4}}-\frac{\omega_{v}}{16} \frac{q^{\prime}(\pi)-q^{\prime}(0)}{s_{k, 1}^{5}}\right. \\
& \left.+O\left(\frac{1}{s_{k, 1}^{6}}\right)\right] \\
& U_{3}\left(y_{v}\left(x, s_{k, 1}\right)\right)=\left(\omega_{v} s_{k, 1}\right)^{2}\left[-\frac{1}{8} \frac{q(\pi)-q(0)}{s_{k, 1}^{4}}-\frac{3 \omega_{v}}{16} \frac{q^{\prime}(\pi)-q^{\prime}(0)}{s_{k, 1}^{5}}\right. \\
& \left.+O\left(\frac{1}{s_{k, 1}^{6}}\right)\right] \text {. }
\end{aligned}
$$

It follows from the conditions $q(\pi)-q(0)=0, q^{\prime}(\pi)-q^{\prime}(0) \neq 0$ that $U_{j+1}\left(y_{v}\left(x, s_{k, 1}\right)\right) \neq 0$ for $j=0,1,2$. Thus, we can seek eigenfunction $y_{k, 1}(x)$ corresponding $\lambda_{k, 1}$ in the form

$$
y_{k, 1}(x)=\left|\begin{array}{cccc}
y_{1}\left(x, s_{k, 1}\right) & y_{2}\left(x, s_{k, 1}\right) & y_{3}\left(x, s_{k, 1}\right) & y_{4}\left(x, s_{k, 1}\right) \\
U_{1}\left(y_{1}\right) & U_{1}\left(y_{2}\right) & U_{1}\left(y_{3}\right) & U_{1}\left(y_{4}\right) \\
U_{2}\left(y_{1}\right) & U_{2}\left(y_{2}\right) & U_{2}\left(y_{3}\right) & U_{2}\left(y_{4}\right) \\
U_{3}\left(y_{1}\right) & U_{3}\left(y_{2}\right) & U_{3}\left(y_{3}\right) & U_{3}\left(y_{4}\right)
\end{array}\right| .
$$

Then,

$$
\begin{aligned}
y_{k, 1}(x)= & y_{1}\left(x, s_{k, 1}\right)\left|\begin{array}{lll}
U_{1}\left(y_{2}\right) & U_{1}\left(y_{3}\right) & U_{1}\left(y_{4}\right) \\
U_{2}\left(y_{2}\right) & U_{2}\left(y_{3}\right) & U_{2}\left(y_{4}\right) \\
U_{3}\left(y_{2}\right) & U_{3}\left(y_{3}\right) & U_{3}\left(y_{4}\right)
\end{array}\right| \\
& -y_{2}\left(x, s_{k, 1}\right)\left|\begin{array}{lll}
U_{1}\left(y_{1}\right) & U_{1}\left(y_{3}\right) & U_{1}\left(y_{4}\right) \\
U_{2}\left(y_{1}\right) & U_{2}\left(y_{3}\right) & U_{2}\left(y_{4}\right) \\
U_{3}\left(y_{1}\right) & U_{3}\left(y_{3}\right) & U_{3}\left(y_{4}\right)
\end{array}\right|
\end{aligned}
$$


Boundary Value Problems

$$
\begin{gathered}
+y_{3}\left(x, s_{k, 1}\right)\left|\begin{array}{lll}
U_{1}\left(y_{1}\right) & U_{1}\left(y_{2}\right) & U_{1}\left(y_{4}\right) \\
U_{2}\left(y_{1}\right) & U_{2}\left(y_{2}\right) & U_{2}\left(y_{4}\right) \\
U_{3}\left(y_{1}\right) & U_{3}\left(y_{2}\right) & U_{3}\left(y_{4}\right)
\end{array}\right| \\
-y_{4}\left(x, s_{k, 1}\right)\left|\begin{array}{lll}
U_{1}\left(y_{1}\right) & U_{1}\left(y_{2}\right) & U_{1}\left(y_{3}\right) \\
U_{2}\left(y_{1}\right) & U_{2}\left(y_{2}\right) & U_{2}\left(y_{3}\right) \\
U_{3}\left(y_{1}\right) & U_{3}\left(y_{2}\right) & U_{3}\left(y_{3}\right)
\end{array}\right| .
\end{gathered}
$$

By simple computations, we have

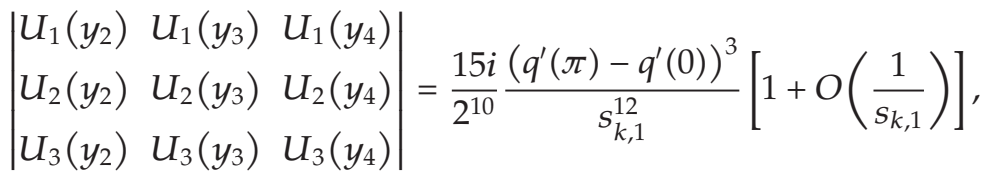

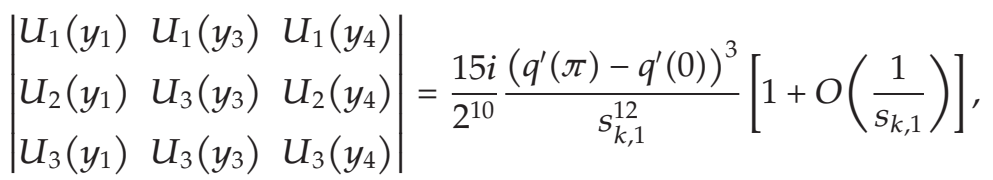

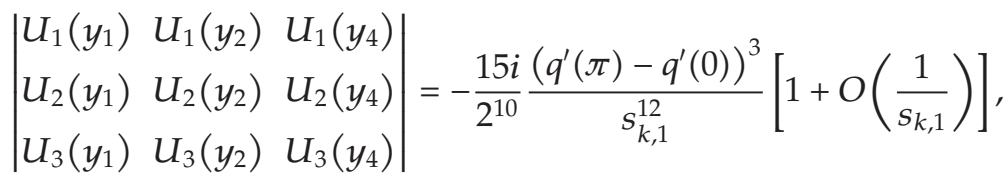

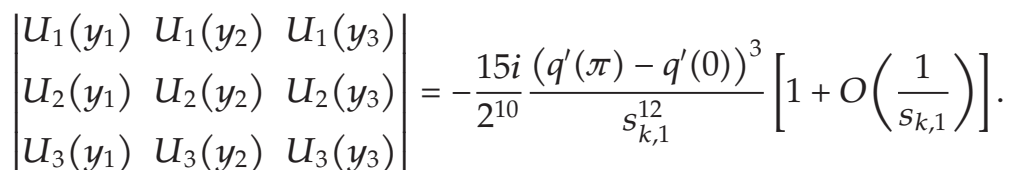

Hence, using the formula (2.2), we can write

$$
\begin{aligned}
y_{k, 1}(x) & =\frac{15 i}{2^{10}} \frac{\left(q^{\prime}(\pi)-q^{\prime}(0)\right)^{3}}{s_{k, 1}^{12}}\left[e^{i s_{k, 1} x}-e^{s_{k, 1} x}-e^{-s_{k, 1} x}+e^{-i s_{k, 1} x}+O\left(\frac{1}{s_{k, 1}}\right)\right] \\
& =\frac{15 i}{2^{9}} \frac{\left(q^{\prime}(\pi)-q^{\prime}(0)\right)^{3}}{s_{k, 1}^{12}}\left[\cos s_{k, 1} x-\cosh s_{k, 1} x+O\left(\frac{1}{s_{k, 1}}\right)\right] .
\end{aligned}
$$

Therefore, for the normalized eigenfunction, we get

$$
y_{k, 1}(x)=\cos s_{k, 1} x-\cosh s_{k, 1} x+O\left(\frac{1}{s_{k, 1}}\right) .
$$


Using the relations (3.3) and (3.12), for sufficiently large integer $k$, we obtain (4.17):

$$
y_{k, 1}(x)=\cos (2 k x)-\cosh (2 k x)+O\left(\frac{1}{k}\right) .
$$

In similar way, we can seek eigenfunction $y_{k, 2}(x)$ corresponding $\lambda_{k, 2}$ in the form

$$
y_{k, 2}(x)=\left|\begin{array}{cccc}
y_{1}\left(x, s_{k, 2}\right) & y_{2}\left(x, s_{k, 2}\right) & y_{3}\left(x, s_{k, 2}\right) & y_{4}\left(x, s_{k, 2}\right) \\
U_{2}\left(y_{1}\right) & U_{2}\left(y_{2}\right) & U_{2}\left(y_{3}\right) & U_{2}\left(y_{4}\right) \\
U_{3}\left(y_{1}\right) & U_{3}\left(y_{2}\right) & U_{3}\left(y_{3}\right) & U_{3}\left(y_{4}\right) \\
U_{4}\left(y_{1}\right) & U_{4}\left(y_{2}\right) & U_{4}\left(y_{3}\right) & U_{4}\left(y_{4}\right)
\end{array}\right| .
$$

Then,

$$
\begin{aligned}
y_{k, 2}(x)= & y_{1}\left(x, s_{k, 2}\right)\left|\begin{array}{lll}
U_{2}\left(y_{2}\right) & U_{2}\left(y_{3}\right) & U_{2}\left(y_{4}\right) \\
U_{3}\left(y_{2}\right) & U_{3}\left(y_{3}\right) & u_{3}\left(y_{4}\right) \\
U_{4}\left(y_{2}\right) & U_{4}\left(y_{3}\right) & u_{4}\left(y_{4}\right)
\end{array}\right| \\
& -y_{2}\left(x, s_{k, 2}\right)\left|\begin{array}{lll}
U_{2}\left(y_{1}\right) & U_{2}\left(y_{3}\right) & U_{2}\left(y_{4}\right) \\
U_{3}\left(y_{1}\right) & U_{3}\left(y_{3}\right) & U_{3}\left(y_{4}\right) \\
U_{4}\left(y_{1}\right) & U_{4}\left(y_{3}\right) & U_{4}\left(y_{4}\right)
\end{array}\right| \\
& +y_{3}\left(x, s_{k, 2}\right)\left|\begin{array}{lll}
U_{2}\left(y_{1}\right) & U_{2}\left(y_{2}\right) & U_{2}\left(y_{4}\right) \\
U_{3}\left(y_{1}\right) & U_{3}\left(y_{2}\right) & U_{3}\left(y_{4}\right) \\
U_{4}\left(y_{1}\right) & U_{4}\left(y_{2}\right) & U_{4}\left(y_{4}\right)
\end{array}\right| \\
& -y_{4}\left(x, s_{k, 2}\right)\left|\begin{array}{lll}
U_{2}\left(y_{1}\right) & U_{2}\left(y_{2}\right) & U_{2}\left(y_{3}\right) \\
U_{3}\left(y_{1}\right) & U_{3}\left(y_{2}\right) & U_{3}\left(y_{3}\right) \\
U_{4}\left(y_{1}\right) & U_{4}\left(y_{2}\right) & U_{4}\left(y_{3}\right)
\end{array}\right| .
\end{aligned}
$$

By simple computations, we get

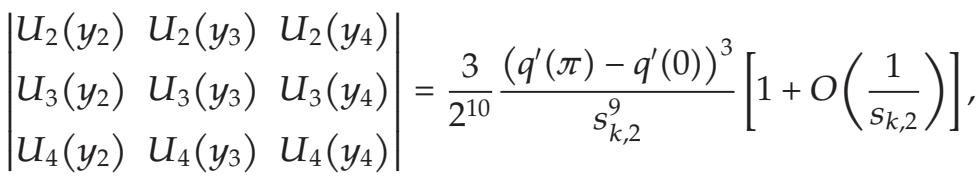

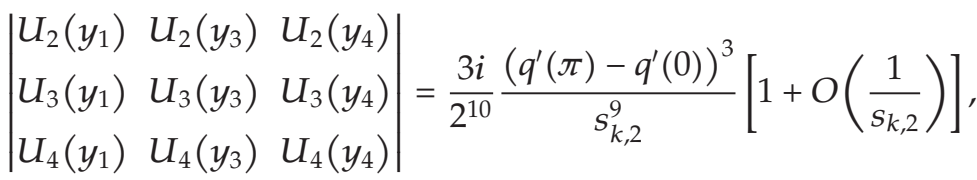




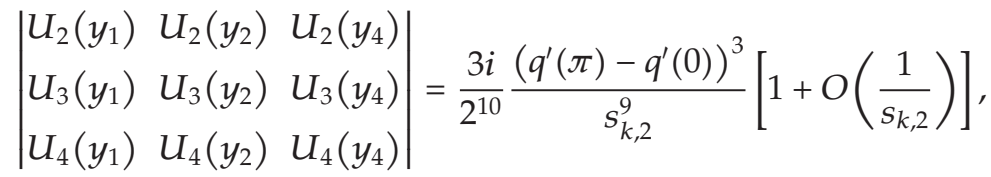

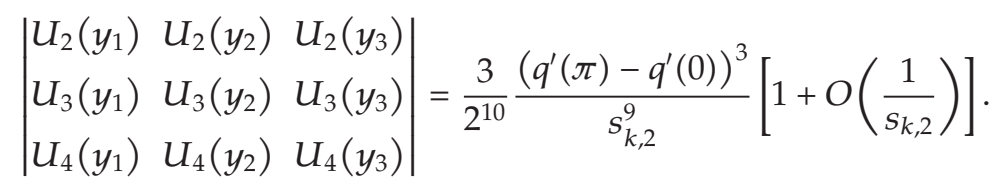

Hence, using the formula (2.2), we can write

$$
\begin{aligned}
y_{k, 2}(x) & =\frac{3}{2^{10}} \frac{\left(q^{\prime}(\pi)-q^{\prime}(0)\right)^{3}}{s_{k, 2}^{9}}\left[e^{i s_{k, 2} x}-i e^{s_{k, 2} x}+i e^{-s_{k, 2} x}-e^{-i s_{k, 2} x}+O\left(\frac{1}{s_{k, 2}}\right)\right] \\
& =\frac{3 i}{2^{9}} \frac{\left(q^{\prime}(\pi)-q^{\prime}(0)\right)^{3}}{s_{k, 2}^{9}}\left[\sin s_{k, 2} x-\sinh s_{k, 2} x+O\left(\frac{1}{s_{k, 2}}\right)\right] .
\end{aligned}
$$

Therefore, for the normalized eigenfunction, we get

$$
y_{k, 2}(x)=\sin s_{k, 2} x-\sinh s_{k, 2} x+O\left(\frac{1}{s_{k, 2}}\right)+O\left(\frac{1}{s_{k, 2}}\right)
$$

Hence, for sufficiently large integer $k$, we obtain (4.18):

$$
y_{k, 2}(x)=\sin (2 k x)-\sinh (2 k x)+O\left(\frac{1}{k}\right)
$$

Case 3. Assume that $q(x) \in C^{(3)}[0, \pi]$ and the conditions $q^{(j)}(\pi)-q^{(j)}(0)=0, j=0,1$ and $q^{\prime \prime}(\pi)-q^{\prime \prime}(0) \neq 0$ hold. Based on the asymptotic expressions of the fundamental solutions of (1.1) and the asymptotic formulas for eigenvalues of the boundary-value problem (1.1), (1.2) up to order $O\left(s^{-7}\right)$, the following result is valid.

Theorem 4.3. If the conditions $q^{(j)}(\pi)-q^{(j)}(0)=0, j=0,1$ and $q^{\prime \prime}(\pi)-q^{\prime \prime}(0) \neq 0$ hold, then eigenfunctions of the boundary-value problem (1.1), (1.2) corresponding the eigenvalues $\lambda_{k, 1}$ and $\lambda_{k, 2}$ are of the form

$$
\begin{aligned}
& y_{k, 1}(x)=\sin (2 k x)+\sinh (2 k x)+O\left(\frac{1}{k}\right), \\
& y_{k, 2}(x)=\cos (2 k x)-\cosh (2 k x)+O\left(\frac{1}{k}\right)
\end{aligned}
$$

where $k$ is sufficiently large integer. 
Proof. It is clear that

$$
\begin{aligned}
& U_{1}\left(y_{v}\left(x, s_{k, 1}\right)\right)=\frac{3}{8} \frac{q(\pi)-q(0)}{s_{k, 1}^{4}}+\frac{5 \omega_{v}}{16} \frac{q^{\prime}(\pi)-q^{\prime}(0)}{s_{k, 1}^{5}}-\frac{5 \omega_{v}^{2}}{32} \frac{q^{\prime \prime}(\pi)-q^{\prime \prime}(0)}{s_{k, 1}^{6}}+O\left(\frac{1}{s_{k, 1}^{7}}\right), \\
& U_{2}\left(y_{v}\left(x, s_{k, 1}\right)\right)=\omega_{v} s_{k, 1}\left\{\frac{1}{8} \frac{q(\pi)-q(0)}{s_{k, 1}^{4}}-\frac{\omega_{v}}{16} \frac{q^{\prime}(\pi)-q^{\prime}(0)}{s_{k, 1}^{5}}-\frac{5 \omega_{v}^{2}}{32} \frac{q^{\prime \prime}(\pi)-q^{\prime \prime}(0)}{s_{k, 1}^{6}}\right. \\
& \left.+O\left(\frac{1}{s_{k, 1}^{7}}\right)\right\} \\
& U_{3}\left(y_{v}\left(x, s_{k, 1}\right)\right)=\left(\omega_{v} s_{k, 1}\right)^{2}\left\{-\frac{1}{8} \frac{q(\pi)-q(0)}{s_{k, 1}^{4}}-\frac{3 \omega_{v}}{16} \frac{q^{\prime}(\pi)-q^{\prime}(0)}{s_{k, 1}^{5}}-\frac{3 \omega_{v}^{2}}{32} \frac{q^{\prime \prime}(\pi)-q^{\prime \prime}(0)}{s_{k, 1}^{6}}\right. \\
& \left.+O\left(\frac{1}{s_{k, 1}^{7}}\right)\right\} \text {. }
\end{aligned}
$$

From the conditions $q^{(j)}(\pi)-q^{(j)}(0)=0(j=0,1)$ and $q^{\prime \prime}(\pi)-q^{\prime \prime}(0) \neq 0$, we have $U_{j+1}\left(y_{v}\left(x, s_{k, 1}\right) \neq 0\right.$ for $j=0,1,2$. Thus, we can seek eigenfunction $y_{k, 1}(x)$ corresponding $\lambda_{k, 1}$ in the form

$$
y_{k, 1}(x)=\left|\begin{array}{cccc}
y_{1}\left(x, s_{k, 1}\right) & y_{2}\left(x, s_{k, 1}\right) & y_{3}\left(x, s_{k, 1}\right) & y_{4}\left(x, s_{k, 1}\right) \\
U_{1}\left(y_{1}\right) & U_{1}\left(y_{2}\right) & U_{1}\left(y_{3}\right) & U_{1}\left(y_{4}\right) \\
U_{2}\left(y_{1}\right) & U_{2}\left(y_{2}\right) & U_{2}\left(y_{3}\right) & U_{2}\left(y_{4}\right) \\
U_{3}\left(y_{1}\right) & U_{3}\left(y_{2}\right) & U_{3}\left(y_{3}\right) & U_{3}\left(y_{4}\right)
\end{array}\right| .
$$

Then,

$$
\begin{aligned}
& y_{k, 1}(x)=y_{1}\left(x, s_{k, 1}\right)\left|\begin{array}{lll}
U_{1}\left(y_{2}\right) & U_{1}\left(y_{3}\right) & U_{1}\left(y_{4}\right) \\
U_{2}\left(y_{2}\right) & U_{2}\left(y_{3}\right) & U_{2}\left(y_{4}\right) \\
U_{3}\left(y_{2}\right) & U_{3}\left(y_{3}\right) & U_{3}\left(y_{4}\right)
\end{array}\right| \\
& -y_{2}\left(x, s_{k, 1}\right)\left|\begin{array}{lll}
U_{1}\left(y_{1}\right) & U_{1}\left(y_{3}\right) & U_{1}\left(y_{4}\right) \\
U_{2}\left(y_{1}\right) & U_{2}\left(y_{3}\right) & U_{2}\left(y_{4}\right) \\
U_{3}\left(y_{1}\right) & U_{3}\left(y_{3}\right) & U_{3}\left(y_{4}\right)
\end{array}\right| \\
& +y_{3}\left(x, s_{k, 1}\right)\left|\begin{array}{lll}
U_{1}\left(y_{1}\right) & U_{1}\left(y_{2}\right) & U_{1}\left(y_{4}\right) \\
U_{2}\left(y_{1}\right) & U_{2}\left(y_{2}\right) & U_{2}\left(y_{4}\right) \\
U_{3}\left(y_{1}\right) & U_{3}\left(y_{2}\right) & U_{3}\left(y_{4}\right)
\end{array}\right| \\
& -y_{4}\left(x, s_{k, 1}\right)\left|\begin{array}{lll}
U_{1}\left(y_{1}\right) & U_{1}\left(y_{2}\right) & U_{1}\left(y_{3}\right) \\
U_{2}\left(y_{1}\right) & U_{2}\left(y_{2}\right) & U_{2}\left(y_{3}\right) \\
U_{3}\left(y_{1}\right) & U_{3}\left(y_{2}\right) & U_{3}\left(y_{3}\right)
\end{array}\right| \text {. }
\end{aligned}
$$


By simple calculations, we get

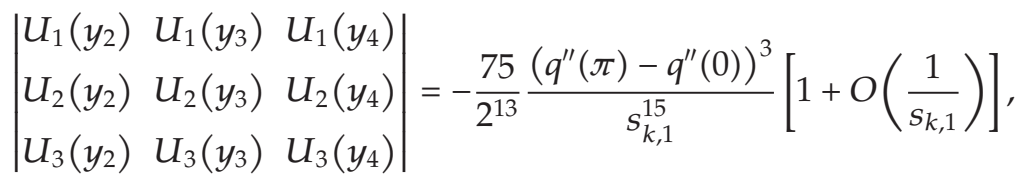

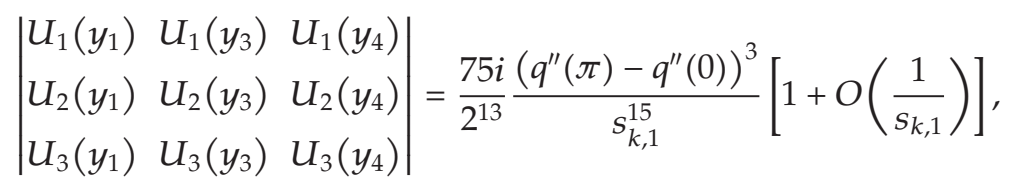

$$
\begin{aligned}
& \left|\begin{array}{lll}
U_{1}\left(y_{1}\right) & U_{1}\left(y_{2}\right) & U_{1}\left(y_{4}\right) \\
U_{2}\left(y_{1}\right) & U_{2}\left(y_{2}\right) & U_{2}\left(y_{4}\right) \\
U_{3}\left(y_{1}\right) & U_{3}\left(y_{2}\right) & U_{3}\left(y_{4}\right)
\end{array}\right|=\frac{75 i}{2^{13}} \frac{\left(q^{\prime \prime}(\pi)-q^{\prime \prime}(0)\right)^{3}}{s_{k, 1}^{15}}\left[1+O\left(\frac{1}{s_{k, 1}}\right)\right],
\end{aligned}
$$

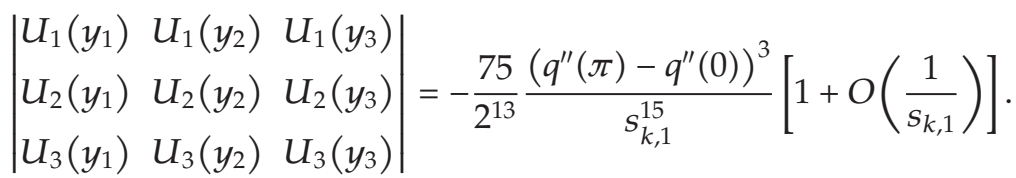

Hence, using the formula (2.2), we can write

$$
\begin{aligned}
y_{k, 1}(x) & =-\frac{75}{2^{13}} \frac{\left(q^{\prime \prime}(\pi)-q^{\prime \prime}(0)\right)^{3}}{s_{k, 1}^{15}}\left[e^{i s_{k, 1} x}+i e^{s_{k, 1} x}-i e^{-s_{k, 1} x}-e^{-i s_{k, 1} x}+O\left(\frac{1}{s_{k, 1}}\right)\right] \\
& =-\frac{75 i}{2^{12}} \frac{\left(q^{\prime \prime}(\pi)-q^{\prime \prime}(0)\right)^{3}}{s_{k, 1}^{15}}\left[\sin s_{k, 1} x+\sinh s_{k, 1} x+O\left(\frac{1}{s_{k, 1}}\right)\right] .
\end{aligned}
$$

Therefore, for the normalized eigenfunction, we get

$$
y_{k, 1}(x)=\sin s_{k, 1} x+\sinh s_{k, 1} x+O\left(\frac{1}{s_{k, 1}}\right) .
$$

Using the relations (3.3) and (3.12), for sufficiently large integer $k$, we obtain (4.32)

$$
y_{k, 1}(x)=\sin (2 k x)+\sinh (2 k x)+O\left(\frac{1}{k}\right) .
$$

In similar way, we can seek eigenfunction $y_{k, 2}(x)$ corresponding $\lambda_{k, 2}$ in the form

$$
y_{k, 2}(x)=\left|\begin{array}{cccc}
y_{1}\left(x, s_{k, 2}\right) & y_{2}\left(x, s_{k, 2}\right) & y_{3}\left(x, s_{k, 2}\right) & y_{4}\left(x, s_{k, 2}\right) \\
U_{2}\left(y_{1}\right) & U_{2}\left(y_{2}\right) & U_{2}\left(y_{3}\right) & U_{2}\left(y_{4}\right) \\
U_{3}\left(y_{1}\right) & U_{3}\left(y_{2}\right) & U_{3}\left(y_{3}\right) & U_{3}\left(y_{4}\right) \\
U_{4}\left(y_{1}\right) & U_{4}\left(y_{2}\right) & U_{4}\left(y_{3}\right) & U_{4}\left(y_{4}\right)
\end{array}\right| .
$$


Then,

$$
\begin{aligned}
& y_{k, 2}(x)=y_{1}\left(x, s_{k, 2}\right)\left|\begin{array}{lll}
U_{2}\left(y_{2}\right) & U_{2}\left(y_{3}\right) & U_{2}\left(y_{4}\right) \\
U_{3}\left(y_{2}\right) & U_{3}\left(y_{3}\right) & U_{3}\left(y_{4}\right) \\
U_{4}\left(y_{2}\right) & U_{4}\left(y_{3}\right) & U_{4}\left(y_{4}\right)
\end{array}\right| \\
& -y_{2}\left(x, s_{k, 2}\right)\left|\begin{array}{lll}
U_{2}\left(y_{1}\right) & U_{2}\left(y_{3}\right) & U_{2}\left(y_{4}\right) \\
U_{3}\left(y_{1}\right) & U_{3}\left(y_{3}\right) & U_{3}\left(y_{4}\right) \\
U_{4}\left(y_{1}\right) & U_{4}\left(y_{3}\right) & U_{4}\left(y_{4}\right)
\end{array}\right| \\
& +y_{3}\left(x, s_{k, 2}\right)\left|\begin{array}{lll}
U_{2}\left(y_{1}\right) & U_{2}\left(y_{2}\right) & U_{2}\left(y_{4}\right) \\
U_{3}\left(y_{1}\right) & U_{3}\left(y_{2}\right) & U_{3}\left(y_{4}\right) \\
U_{4}\left(y_{1}\right) & U_{4}\left(y_{2}\right) & U_{4}\left(y_{4}\right)
\end{array}\right| \\
& -y_{4}\left(x, s_{k, 2}\right)\left|\begin{array}{lll}
U_{2}\left(y_{1}\right) & U_{2}\left(y_{2}\right) & U_{2}\left(y_{3}\right) \\
U_{3}\left(y_{1}\right) & U_{3}\left(y_{2}\right) & U_{3}\left(y_{3}\right) \\
U_{4}\left(y_{1}\right) & U_{4}\left(y_{2}\right) & U_{4}\left(y_{3}\right)
\end{array}\right| .
\end{aligned}
$$

By simple computations, we get

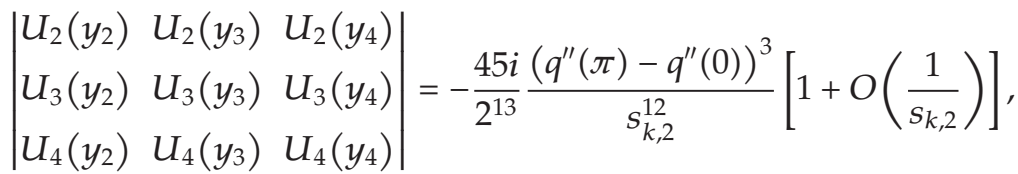

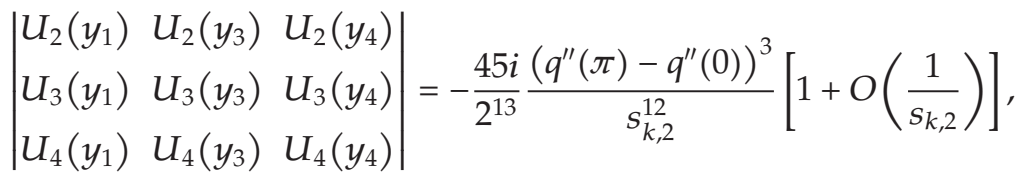

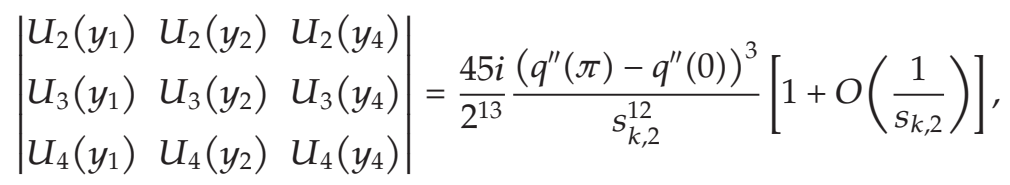

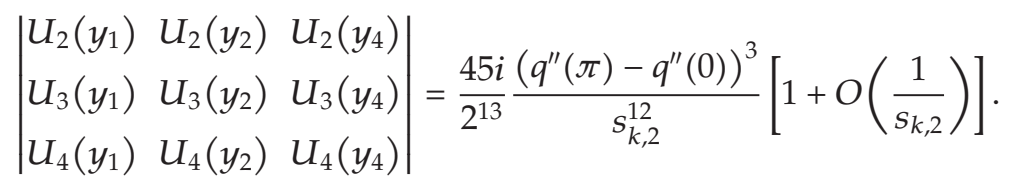

By the formula (2.2), we can write

$$
\begin{aligned}
y_{k, 2}(x) & =-\frac{45 i}{2^{13}} \frac{\left(q^{\prime \prime}(\pi)-q^{\prime \prime}(0)\right)^{3}}{s_{k, 2}^{12}}\left[e^{i s_{k, 2} x}-e^{s_{k, 2} x}-e^{-s_{k, 2} x}+e^{-i s_{k, 2} x}+O\left(\frac{1}{s_{k, 2}}\right)\right] \\
& =\frac{45 i}{2^{12}} \frac{\left(q^{\prime \prime}(\pi)-q^{\prime \prime}(0)\right)^{3}}{s_{k, 2}^{12}}\left[\cos s_{k, 2} x-\cosh s_{k, 2} x+O\left(\frac{1}{s_{k, 2}}\right)\right] .
\end{aligned}
$$


Therefore, for the normalized eigenfunction, we get

$$
y_{k, 2}(x)=\cos s_{k, 2} x-\cosh s_{k, 2} x+O\left(\frac{1}{s_{k, 2}}\right) .
$$

Hence, for sufficiently large integer $k$, we obtain the relation (4.33)

$$
y_{k, 2}(x)=\cos (2 k x)-\cosh (2 k x)+O\left(\frac{1}{k}\right) .
$$

Case 4. Assume that $q(x) \in C^{(4)}[0, \pi]$ and the conditions $q^{(j)}(\pi)-q^{(j)}(0)=0, j=\overline{0,2}$ and $q^{\prime \prime \prime}(\pi)-q^{\prime \prime \prime}(0) \neq 0$ hold. Based on the asymptotic expressions of the fundamental solutions of (1.1) and the asymptotic formulas for eigenvalues of the boundary-value problem (1.1), (1.2) up to order $O\left(s^{-8}\right)$, the following result is valid.

Theorem 4.4. If the conditions $q^{(j)}(\pi)-q^{(j)}(0)=0, j=\overline{0,2}$ and $q^{\prime \prime \prime}(\pi)-q^{\prime \prime \prime}(0) \neq 0$ hold, then eigenfunctions of the boundary-value problem (1.1), (1.2) corresponding the eigenvalues $\lambda_{k, 1}$ and $\lambda_{k, 2}$ are of the form

$$
\begin{aligned}
& y_{k, 1}(x)=\cos (2 k x)+\cosh (2 k x)+O\left(\frac{1}{k}\right), \\
& y_{k, 2}(x)=\sin (2 k x)+\sinh (2 k x)+O\left(\frac{1}{k}\right),
\end{aligned}
$$

where $k$ is sufficiently large integer.

Proof. It is clear that

$$
\begin{aligned}
U_{1}\left(y_{v}\left(x, s_{k, 1}\right)\right)= & \frac{3}{8} \frac{q(\pi)-q(0)}{s_{k, 1}^{4}}+\frac{5 \omega_{v}}{16} \frac{q^{\prime}(\pi)-q^{\prime}(0)}{s_{k, 1}^{5}} \\
& -\frac{5 \omega_{v}^{2}}{32} \frac{q^{\prime \prime}(\pi)-q^{\prime \prime}(0)}{s_{k, 1}^{6}}-\frac{\omega_{v}}{64} \frac{q^{\prime \prime \prime}(\pi)-q^{\prime \prime \prime}(0)}{s_{k, 1}^{7}}+O\left(\frac{1}{s_{k, 1}^{8}}\right), \\
U_{2}\left(y_{v}\left(x, s_{k, 1}\right)\right)= & \omega_{v} s_{k, 1}\left\{\frac{1}{8} \frac{q(\pi)-q(0)}{s_{k, 1}^{4}}-\frac{\omega_{v}}{16} \frac{q^{\prime}(\pi)-q^{\prime}(0)}{s_{k, 1}^{5}}\right. \\
& \left.\quad-\frac{5 \omega_{v}^{2}}{32} \frac{q^{\prime \prime}(\pi)-q^{\prime \prime}(0)}{s_{k, 1}^{6}}+\frac{9 \omega_{v}}{64} \frac{q^{\prime \prime \prime}(\pi)-q^{\prime \prime \prime}(0)}{s_{k, 1}^{7}}+O\left(\frac{1}{s_{k, 1}^{8}}\right)\right\},
\end{aligned}
$$




$$
\begin{aligned}
U_{3}\left(y_{v}\left(x, s_{k, 1}\right)\right)=\left(\omega_{v} s_{k, 1}\right)^{2}\{ & -\frac{1}{8} \frac{q(\pi)-q(0)}{s_{k, 1}^{4}}-\frac{3 \omega_{v}}{16} \frac{q^{\prime}(\pi)-q^{\prime}(0)}{s_{k, 1}^{5}} \\
& \left.-\frac{3 \omega_{v}^{2}}{32} \frac{q^{\prime \prime}(\pi)-q^{\prime \prime}(0)}{s_{k, 1}^{6}}-\frac{\omega_{v}}{64} \frac{q^{\prime \prime \prime}(\pi)-q^{\prime \prime \prime}(0)}{s_{k, 1}^{7}}+O\left(\frac{1}{s_{k, 1}^{8}}\right)\right\} .
\end{aligned}
$$

From the conditions $q^{(j)}(\pi)-q^{(j)}(0)=0(j=\overline{0,2})$ and $q^{\prime \prime \prime}(\pi)-q^{\prime \prime \prime}(0) \neq 0$, we have $U_{j+1}\left(y_{v}\left(x, s_{k, 1}\right) \neq 0\right.$ for $j=0,1,2$. Thus, we can seek eigenfunction $y_{k, 1}(x)$ corresponding $\lambda_{k, 1}$ in the form

$$
y_{k, 1}(x)=\left|\begin{array}{cccc}
y_{1}\left(x, s_{k, 1}\right) & y_{2}\left(x, s_{k, 1}\right) & y_{3}\left(x, s_{k, 1}\right) & y_{4}\left(x, s_{k, 1}\right) \\
U_{1}\left(y_{1}\right) & U_{1}\left(y_{2}\right) & U_{1}\left(y_{3}\right) & U_{1}\left(y_{4}\right) \\
U_{2}\left(y_{1}\right) & U_{2}\left(y_{2}\right) & U_{2}\left(y_{3}\right) & U_{2}\left(y_{4}\right) \\
U_{3}\left(y_{1}\right) & U_{3}\left(y_{2}\right) & U_{3}\left(y_{3}\right) & U_{3}\left(y_{4}\right)
\end{array}\right| .
$$

Then

$$
\begin{aligned}
y_{k, 1}(x)= & y_{1}\left(x, s_{k, 1}\right)\left|\begin{array}{lll}
U_{1}\left(y_{2}\right) & U_{1}\left(y_{3}\right) & U_{1}\left(y_{4}\right) \\
U_{2}\left(y_{2}\right) & U_{2}\left(y_{3}\right) & u_{2}\left(y_{4}\right) \\
U_{3}\left(y_{2}\right) & U_{3}\left(y_{3}\right) & U_{3}\left(y_{4}\right)
\end{array}\right| \\
& -y_{2}\left(x, s_{k, 1}\right)\left|\begin{array}{lll}
U_{1}\left(y_{1}\right) & U_{1}\left(y_{3}\right) & U_{1}\left(y_{4}\right) \\
U_{2}\left(y_{1}\right) & U_{2}\left(y_{3}\right) & U_{2}\left(y_{4}\right) \\
U_{3}\left(y_{1}\right) & U_{3}\left(y_{3}\right) & U_{3}\left(y_{4}\right)
\end{array}\right| \\
& +y_{3}\left(x, s_{k, 1}\right)\left|\begin{array}{lll}
U_{1}\left(y_{1}\right) & U_{1}\left(y_{2}\right) & U_{1}\left(y_{4}\right) \\
U_{2}\left(y_{1}\right) & U_{2}\left(y_{2}\right) & U_{2}\left(y_{4}\right) \\
U_{3}\left(y_{1}\right) & U_{3}\left(y_{2}\right) & U_{3}\left(y_{4}\right)
\end{array}\right| \\
& -y_{4}\left(x, s_{k, 1}\right)\left|\begin{array}{lll}
U_{1}\left(y_{1}\right) & U_{1}\left(y_{2}\right) & U_{1}\left(y_{3}\right) \\
U_{2}\left(y_{1}\right) & U_{2}\left(y_{2}\right) & U_{2}\left(y_{3}\right) \\
U_{3}\left(y_{1}\right) & U_{3}\left(y_{2}\right) & U_{3}\left(y_{3}\right)
\end{array}\right| .
\end{aligned}
$$

By simple computations, we get

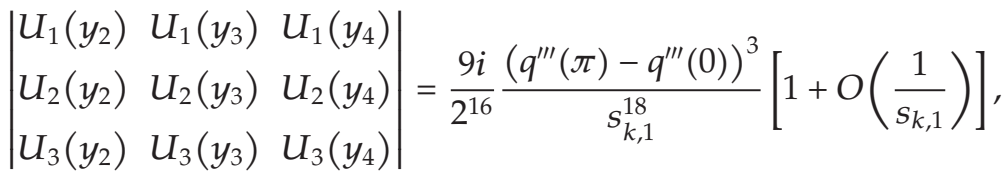

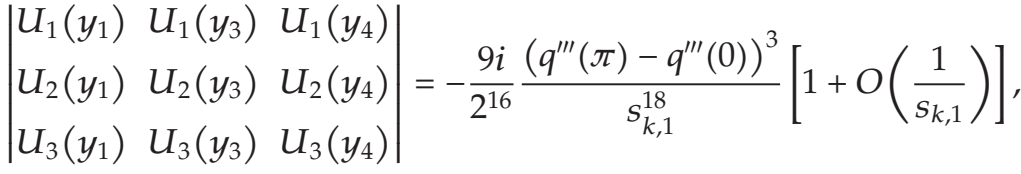


Boundary Value Problems

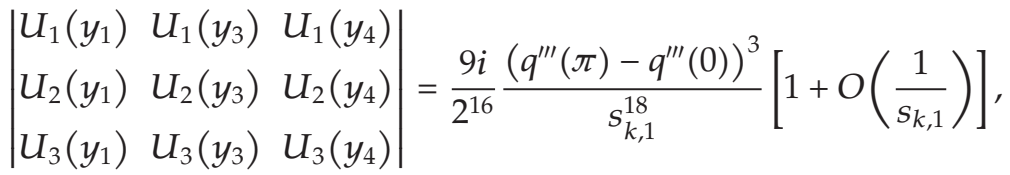

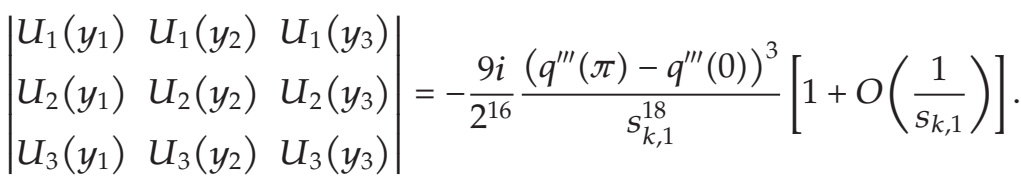

By the formula (2.2), we can write

$$
\begin{aligned}
y_{k, 1}(x) & =\frac{9 i}{2^{16}} \frac{\left(q^{\prime \prime \prime}(\pi)-q^{\prime \prime \prime}(0)\right)^{3}}{s_{k, 1}^{18}}\left[e^{i s_{k, 1} x}+e^{s_{k, 1} x}+e^{-s_{k, 1} x}+e^{-i s_{k, 1} x}+O\left(\frac{1}{s_{k, 1}}\right)\right] \\
& =\frac{9 i}{2^{15}} \frac{\left(q^{\prime \prime \prime}(\pi)-q^{\prime \prime \prime}(0)\right)^{3}}{s_{k, 1}^{18}}\left[\cos s_{k, 1} x+\cosh s_{k, 1} x+O\left(\frac{1}{s_{k, 1}}\right)\right] .
\end{aligned}
$$

Therefore, for the normalized eigenfunction, we get

$$
y_{k, 1}(x)=\cos s_{k, 1} x+\cosh s_{k, 1} x+O\left(\frac{1}{s_{k, 1}}\right) .
$$

Using the relations (3.3) and (3.12), for sufficiently large integer $k$, we obtain (4.47):

$$
y_{k, 1}(x)=\cos (2 k x)+\cosh (2 k x)+O\left(\frac{1}{k}\right) .
$$

In similar way, we can seek eigenfunction $y_{k, 2}(x)$ corresponding $\lambda_{k, 2}$ in the form

$$
y_{k, 2}(x)=\left|\begin{array}{cccc}
y_{1}\left(x, s_{k, 2}\right) & y_{2}\left(x, s_{k, 2}\right) & y_{3}\left(x, s_{k, 2}\right) & y_{4}\left(x, s_{k, 2}\right) \\
U_{2}\left(y_{1}\right) & U_{2}\left(y_{2}\right) & U_{2}\left(y_{3}\right) & U_{2}\left(y_{4}\right) \\
U_{3}\left(y_{1}\right) & U_{3}\left(y_{2}\right) & U_{3}\left(y_{3}\right) & U_{3}\left(y_{4}\right) \\
U_{4}\left(y_{1}\right) & U_{4}\left(y_{2}\right) & U_{4}\left(y_{3}\right) & U_{4}\left(y_{4}\right)
\end{array}\right| .
$$


By simple computations, we get

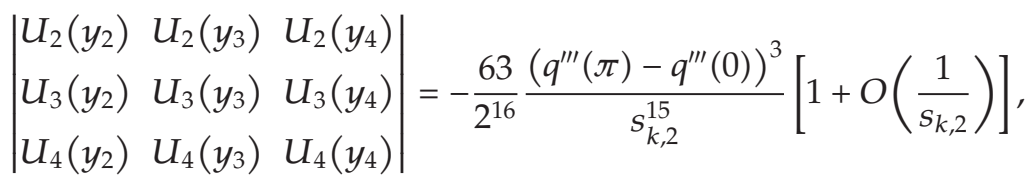

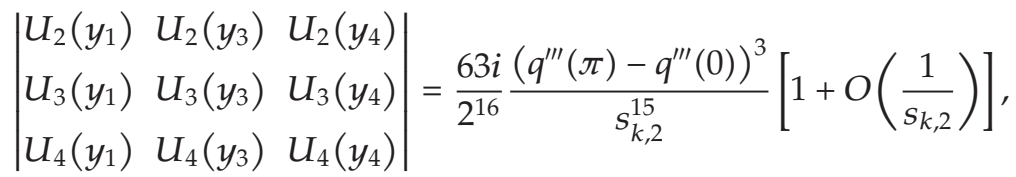

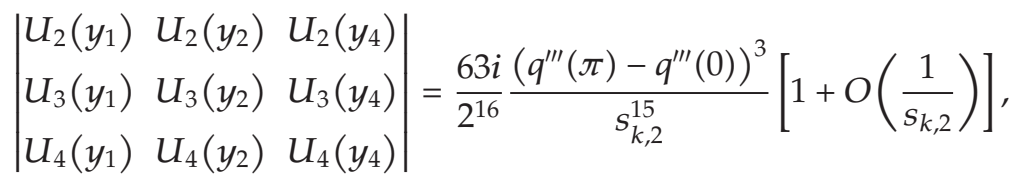

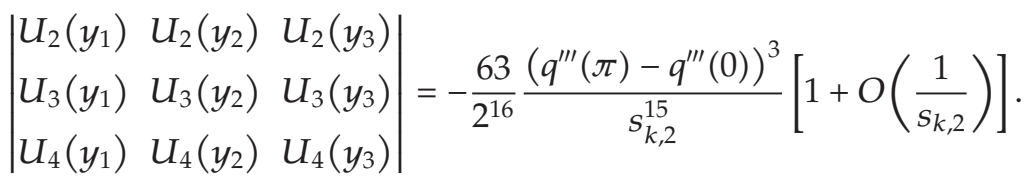

By the formula (2.2), we can write

$$
\begin{aligned}
y_{k, 2}(x) & =-\frac{63}{2^{16}} \frac{\left(q^{\prime \prime \prime}(\pi)-q^{\prime \prime \prime}(0)\right)^{3}}{s_{k, 2}^{15}}\left[e^{i s_{k, 2} x}+i e^{s_{k, 2} x}-i e^{-s_{k, 2} x}-e^{-i s_{k, 2} x}+O\left(\frac{1}{s_{k, 2}}\right)\right] \\
& =-\frac{63 i}{2^{15}} \frac{\left(q^{\prime \prime \prime}(\pi)-q^{\prime \prime \prime}(0)\right)^{3}}{s_{k, 2}^{15}}\left[\sin s_{k, 2} x+\sinh s_{k, 2} x+O\left(\frac{1}{s_{k, 2}}\right)\right] .
\end{aligned}
$$

Therefore, for the normalized eigenfunction, we get

$$
y_{k, 2}(x)=\sin s_{k, 2} x+\sinh s_{k, 2} x+O\left(\frac{1}{s_{k, 2}}\right) .
$$

Hence, for sufficiently large integer $k$, we obtain the relation (4.48)

$$
y_{k, 2}(x)=\sin (2 k x)+\sinh (2 k x)+O\left(\frac{1}{k}\right) .
$$

\section{Acknowledgments}

This work is supported by The Scientific and Technological Research Council of Turkey (TÜBITAK). The author would like to thank the referee and the editor for their helpful comments and suggestions. The author also would like to thank prof. Kh. R. Mamedov for useful discussions. 


\section{References}

[1] N. Dunford and J. T. Schwartz, Linear Operators, Part 3 Spectral Operators, Wiley Classics Library, John Wiley \& Sons, New York, NY, USA, 1970.

[2] B. M. Levitan and I. S. Sargsjan, Introduction to Spectral Theory: Selfadjoint Ordinary Differential Operators, American Mathematical Society, Providence, RI, USA, 1975.

[3] V. A. Marchenko, Sturm-Liouville Operators and Applications, vol. 22 of Operator Theory: Advances and Applications, Birkhäuser, Basel, Switzerland, 1986.

[4] M. A. Naimark, Linear Differential Operators. Part 1, Frederick Ungar, New York, NY, USA, 1967.

[5] F. S. Rofe-Beketov and A. M. Kholkin, Spectral Analysis of Differential Operators, vol. 7 of World Scientific Monograph Series in Mathematics, World Scientific Publishing, Singapore, 2005.

[6] N. B. Kerimov and K. R. Mamedov, "On the Riesz basis property of root functions of some regular boundary value problems," Mathematical Notes, vol. 64, no. 4, pp. 483-487, 1998.

[7] V. A. Chernyatin, "Higher-order spectral asymptotics for the Sturm-Liouville operator," Ordinary Differential Equations, vol. 38, no. 2, pp. 217-227, 2002.

[8] K. R. Mamedov and H. Menken, "On the basisness in $L_{2}(0,1)$ of the root functions in not strongly regular boundary value problems," European Journal of Pure and Applied Mathematics, vol. 1, no. 2, pp 51-60, 2008.

[9] K. R. Mamedov and H. Menken, "Asymptotic formulas for eigenvalues and eigenfunctions of a nonselfadjoint Sturm-Liouville operator," in Further Progress in Analysis, H. G. W. Begehr, A. O. Çelebi, and R. P. Gilbert, Eds., pp. 798-805, World Scientific Publishing, 2009.

[10] V. M. Kurbanov, "A theorem on equivalent bases for a differential operator," Doklady Akademii Nauk, vol. 406, no. 1, pp. 17-20, 2006.

[11] H. Menken and K. R. Mamedov, "Basis property in $L_{p}(0,1)$ of the root functions corresponding to a boundary-value problem," Journal of Applied Functional Analysis, vol. 5, no. 4, pp. 351-356, 2010. 